\title{
MESURES CONIQUES SUR UN ESPACE DE BANACH OU SON DUAL
}

\author{
RICHARD BECKER
}

(Received 7 October 1983)

Communicated by G. Brown

\begin{abstract}
Si $E$ et $F$ sont deux espaces vectoriels en dualité séparante, $M^{+}(E, F)$ désigne le cône des mesures coniques positives sur $E$ mis en dualité avec $F$, c'est à dire le cônes des formes postives sur le treillis de fonctions sur $E$ engendré par $F$. Ce sont des objets plus généraux que les mesures cylindriques admettant des moments finis d'ordre un.

On part d'abord d'une mesure conique représentée par une mesure de Radon sur le complété faible de $E$ et on donne des critéres (par exemple R.N.P.) pour qu'elle le soit sur l'espace $E$ lui-même.

On étudie ensuite les cônes faiblement complets saillants (classe $\mathscr{S}$ ) contenus dans un espace de Banach ou dans le dual d'un espace de Fréchet $F$; on montre notamment qu' un cône faiblement fermé contenu dans $F^{\prime}$ est dans $\mathscr{S}$ si son polaire dans $F$ est positivement engendré.

Si $B$ est un espace de Banach et $l_{1} \not \subset B$, on cherche à prologner une $\mu \in M^{+}\left(B^{\prime}, B\right)$ en un élement de $M^{+}\left(B^{\prime}, B^{\prime \prime}\right)$. On montre également que, si $X$ est un convexe compact, toute fonction vérifiant le calcul barycentrique sur $X$ est continue sur des ensembles fixes que l'on précise.

Enfin on donne des conditions (de type bornologique) sur un e.l.c.s $E$, permettant d'interpréter une $\mu \in M^{+}\left(E, E^{\prime}\right)$ comme une mesure conique sur un espace normé.
\end{abstract}

1980 Mathematics subject classification (Amer. Math. Soc.): primary 46 B 20, 46 B 22; secondary 28 C $05,28 \mathrm{C} 20$.

\section{Preliminaires}

Rappelons d'abord la définition des mesures coniques ([3], Section 30.38.40): soit $E$ et $F$ deux espaces vectoriels en dualité séparante; on note $h(E, F)$ le treillis de fonctions sur $E$ engendré par $F$; on désigne par $M^{+}(E, F)$ le cône des formes positives sur $h(E, F)$; une $\mu \in M^{+}(E, F)$ est appelée une mesure conique positive sur $E$.

(c) 1985 Australian Mathematical Society $0263-6115 / 85 \$ A 2.00+0.00$ 
On note $r(\mu)$ l'élément de $F^{*}$ (dual algébrique de $F$ ) tel que $\mu(l)=l(r(\mu)$ ) pour toute $l \in F$ et on pose

$$
K_{\mu}=\{r(\lambda): 0 \leqslant \lambda \leqslant \mu\} \subset F^{*}
$$

Enfin, on dit que $\mu$ est localisable dans $E$ lorsqu'il existe une mesure de Radon généralisée ([13], Part I, Chapitre 1, Paragraphe 2) sur $E \backslash 0$ muni de $\sigma(E, F)$ qui représente $\mu$. On sait ([3], 39.4) que les mesures coniques sont des objets plus généraux que les mesures cylindriques admettant des moments d'ordre un finis, à double titre:

(1) on s'affranchit d'une condition de $\sigma$-additivité;

(2) une mesure conique n'est pas nécessairement portée par un hyperplan.

Ce travail comporte quatre parties:

Partie I (Section 1 à 4): on part d'une mesure conique localisable dans un complèté faible et on donne des critères pour qu'elle le soitdans 1'espace de départ lui-même.

Partie II (Section 5 à 10): on étudie les cônes saillants faiblement complets contenus dans un espace de Banach ou dans le dual d'un espace de Fréchet.

Partie III (Section 11 à 20): si $B$ est un espace de Banach, on cherche à prolonger une $\mu \in M^{+}\left(B^{\prime}, B\right)$ en un élément de $M^{+}\left(B^{\prime}, B^{\prime \prime}\right)$.

Partie IV (Section 21 à 25): on donne des conditions permettant d'interpréter une mesure conique sur un e.l.c.s. comme une mesure conique sur un espace normé.

\section{Mesures coniques localisables dans un complété faible}

On dit qu'une forme $T \geqslant 0$ sur un espace vectoriel de fonctions $E$ définies surun ensemble $X$ est une intégrale de Daniell lorsque, pour toute suite $\left(f_{n}\right)$ de $E$, qui décroit vers 0 sur $X$, on a $T\left(f_{n}\right) \rightarrow 0$. Pour la propriété de Radon-Nikodym (R.N.P.) on renvoit à ([2], Chapitre 6, Section 3).

1. Proposition. Soit $B$ un espace de Banach qui possède R.N.P. et $\mu \in$ $M^{+}\left(B, B^{\prime}\right)$ telle que $K_{\mu} \subset B$. Si $\mu$ est localisable dans $\left(B^{\prime}\right)^{*}$, alors $\mu$ est une intégrale de Daniell sur $B$. Si, de plus, $B$ est séparable, alors $\mu$ est localisable dans $B$.

Preuve. Il existe $f \in B^{\prime}$ et $\lambda \leqslant \mu$ tels que $\lambda \neq 0$ soit localisable sur un convexe compact $X$ de $\left(E^{\prime}\right)^{*}$, contenu dans $f^{-1}(1)$, par une mesure de Radon $m \geqslant 0$ de masse 1 .

L'application $\pi$ qui, à tout borélien $A \subset X$ fait correspondre le résultant de la mesure conique localisée par $\left.m\right|_{A}$ est une mesure vectorielle à valeurs dans $B$, 
puisque $K_{\mu} \subset B$. De plus, en posant $a=\pi(A) / m(A)$, on $a: f(a)=$ $f\left(r\left(\left.m\right|_{A}\right)\right) / m(A)=1$, puisque $f=1$ sur $X$. Si $Y=X \cap B$, on a donc $a \in Y$; puisque $B$ possède R.N.P. ([2], Chapitre 6, Section 3) il existe $\varphi: X \rightarrow Y$ Bochner mesurable telle que $\pi(A)=\int_{A} \varphi d m$. Pour toute $h \in h\left(B, B^{\prime}\right)$, de la forme $h=$ $\bigvee_{1 \leqslant i \leqslant n} l_{i}$, où $l_{i} \in B^{\prime}$, on vérifie facilement que $\lambda(h)=\int_{X} h \circ \varphi d m$, d'ou la même formule pour toute $h \in h\left(B, B^{\prime}\right)$, donc $\lambda$ est une intégrale de Daniell sur $B$.

Lorsque $B$ est séparable, alors $Y$ est souslinien et $\lambda$ sera représentée par une mesure de Radon sur $Y$ ([13], Chapitre II, Lemme 18 et Théorem 40). D'où la proposition.

2. Remarque. Soit $B=c_{0}(N)$ et $\mu=\sum_{1}^{\infty} \varepsilon_{e_{n}}$, où $\left(e_{n}\right)_{n \in N}$ est la base canonique de $B$. On voit que $K_{\mu}$ n'est pas contenu dans $B$, bien que $\mu$ soit localisable dans $B$.

Voici deux résultats concernant les mesures coniques sur le dual d'un espace tonnelé.

3. Proposition. Soit $E$ un espace tonnelé de dual $E^{\prime}$ et $\mu \in M^{+}\left(E^{\prime}, E\right)$; si $K_{\mu} \subset E^{\prime}$, alors les deux propriétés suivantes sont équaliventes:

(a) $\mu$ est localisable dans $E^{\prime}$ muni de $\sigma\left(E^{\prime}, E\right)$.

(b) $\mu$ est localisable dans $E^{*}$ muni de $\sigma\left(E^{*}, E\right)$.

Preuve. Il faut montrer que (b) $\Rightarrow$ (a). Il existe $f \in E$ et $\lambda \leqslant \mu$ tels que $\lambda \neq 0$ soit localisable sur un convexe compact $A$ de $E^{*}$, contenu dans $f^{-1}(1)$. L'ensemble $B=\{r(\nu) / \nu(f): 0 \leqslant \nu \leqslant \lambda, \nu \neq 0\}$ est contenu dans $A \cap E^{\prime}$ et est donc $\sigma\left(E^{\prime}, E\right)$ borné; comme $E$ est tonnelé, $B$ est donc relativement compact dans $E^{\prime}$ pour $\sigma\left(E^{\prime}, E\right)$. Comme $\lambda$ est limite de décompositions $\sum_{1}^{n} \varepsilon_{r\left(v_{i}\right)}$ avec $\lambda=\sum_{1}^{n} \nu_{i}$, ([3], 30.9) on voit que $\lambda=\lim \sum_{1}^{n} \alpha_{i} \varepsilon_{a_{i}}$ avec $a_{i}=r\left(\nu_{i}\right) / \nu_{i}(f)$ et $\alpha_{i}=\nu_{i}(f)$; comme $a_{i} \in B$ et que $\sum_{1}^{n} \alpha_{i}=\lambda(f)$, on aura bien localisation de $\lambda$, donc de $\mu$.

4. Proposition. Soit $E$ un espace tonnelé de dual $E^{\prime}$ et $\mu \in M^{+}\left(E^{\prime}, E\right)$; si $\mu$ est localisable dans $E^{\prime}$, alors on a $K_{\mu} \subset E^{\prime}$.

Preuve. Il suffit de montrer que $r(\mu) \in E^{\prime}$. Soit $\left(A_{i}\right)_{i \in I}$ une famille de parties convexes $\sigma\left(X^{\prime}, X\right)$ compactes de $X^{\prime}$ et $\left(m_{i}\right)_{i \in I}$ une famille de mesures de Radon finies, $m_{i}$ étant portée par $A_{i}$, de sorte que $\mu=\sum m_{i}$. Si $f \in E$ et si $J \subset I$ est fini, on $a$ :

$$
\left|\left(\sum_{i \in J} r\left(m_{i}\right)\right)(f)\right| \leqslant \sum_{i \in J}\left|r\left(m_{i}\right)(f)\right| \leqslant \sum_{i \in J} m_{i}(|f|) \leqslant \mu(|f|) .
$$

Comme $E$ est tonnelé, il existe une partie $\sigma\left(E^{\prime}, E\right)$ compacte de $E^{\prime}$ qui contient, pour toute partie $J$ finie de $I$, l'élément $\sum_{i \in J} r\left(m_{i}\right)$, d'où $r(\mu) \in E^{\prime}$. 


\section{Cones convexes saillants faiblement complets contenus} dans un espace de Banach ou le dual d'un espace de Fréchet

La Proposition 3 suggère les résultats suivants concernant les cônes convexes saillants faiblement complets (classe $\mathscr{S}$ ).

5. Proposition. Soit $\Gamma$ un cône convexe contenu dans le dual $F^{\prime} d$ 'un espace de Fréchet $F$; on suppose que tout élément de $F$ est différence de deux éléments (de $F$ ) positifis sur $\Gamma$ et que $\Gamma$ est $\sigma\left(F^{\prime}, F\right)$ fermé. Alors $\Gamma$ est $\sigma\left(F^{\prime}, F\right)$ complet.

Preuve. Soit $\Gamma^{0}$ le polaire de $\Gamma$ dans $F$ et $N=\Gamma^{0} \cap\left(-\Gamma^{0}\right)$; soit $q$ l'application canoniquede $F$ dans $F / N$; on vérifiequedans $F / N$ le cône $q\left(\Gamma^{0}\right)$ est faiblement fermé; comme ce cône engendre $F / N$ et est saillant, on voit que $\Gamma$ s'identifie au cône des formes linéaires continues $\geqslant 0$ sur le Fréchet $F / N$, ordonné par le cône $q\left(\Gamma^{0}\right)$.

Le cône $\Gamma$ est donc faiblement complet pour la dualité avec $F / N$, d'après le théorème de Klee ([12], V.5.5). D’où le résultat.

Voici une conséquence du résultat précédent, dans le cadre des espaces de Banach.

6. Proposition. Soit $\Gamma$ un cône convexe contenu dans un espace de Banach $B$, tel que tout élément de $B^{\prime}$ soit différence de deux éléments (de $B^{\prime}$ ) positifs sur $\Gamma$; les deux propriétés suivantes sont équivalentes:

(1) la trace de $\Gamma$ sur la boule unité fermée de $B$ est $\sigma\left(B, B^{\prime}\right)$ compacte.

(2) $\Gamma$ est $\sigma\left(B, B^{\prime}\right)$ complet.

Preuve. (2) $\Rightarrow(1)$. Il est bien connu ([3], 30.10) qu'une partie bornée et fermée d'un cône de la classe $\mathscr{S}$ est faiblement compacte.

$(1) \Rightarrow(2)$. On plonge $B$ dans $B^{\prime \prime}$; d'après le théorème de Krein-Smulian ([11], page 125) le cône $\Gamma$ est $\sigma\left(B^{\prime \prime}, B^{\prime}\right)$ fermé; il suffit alors d'appliquer le résultat précédent avec $F=B^{\prime}$.

Dans le cadre de la Proposition 5, le cône $\Gamma$ est un $K_{\sigma}$, réunion dénombrable des $\Gamma \cap V_{n}^{0}$, où $\left(V_{n}\right)_{n \in N}$ est une base de voisinages de 0 dans $F$; rappelons alors le résultat suivant, concernant ces cônes, dû à Fakhoury et Rogalsky ([4], 2.4.A).

7. Proposition. Soit $\Gamma \in \mathscr{S}$ un cône recouvert par une suite $\left(K_{n}\right)$ de parties convexes faiblement compactes de $\Gamma$. Alors $\Gamma$ est encore faiblement complet pour la 
dualité avec l'espace $A\left(\left(K_{n}\right)\right)$ des fonctions affines sur $\Gamma$, nulles en 0 , et (de restriction) continues sur chaque $K_{n}$.

Dans le cadre de la proposition précédente, le Lemme 4 de [1] nous permet d'énoncer.

8. Proposition. Soit $K$ une partie compacte de $\Gamma$, pour la dualité de départ; alors $K$ est aussi compacte pour la dualité avec $A\left(\left(K_{n}\right)\right)$.

9. Corollaire. L'espace $A\left(\left(K_{n}\right)\right)$ est indépendant de la suite $\left(K_{n}\right)$ qui recouve $\Gamma$, et c'est le plus grand espace de fonctions affines sur $\Gamma$, nulles en 0 , rendant $\Gamma$ faiblement complet, pour une structure uniforme faible plus fine que celle de départ.

Replacons-nous maintenant dans le cadre de la Proposition 5, en supposant de plus que le polaire $\Gamma^{0}$ de $\Gamma$ dans $F$ soit saillant. On note $A$ l'espace $A\left(\left(\Gamma \cap V_{n}^{0}\right)_{n \in M}\right)$; il est clair que $F$ s'identifie algébriquement à un sous-espace de $A$. La notion de cône normal figure dans ([12], V.3).

10. PROPOSITION. Les trois propriétés suivantes sont équivalentes:

(1) $F=A$.

(2) Le cône $\Gamma^{0}$ est normal dans $F$.

(3) $F^{\prime}=\Gamma-\Gamma$.

Preuve. (1) $\Rightarrow$ (2). Si $F=A$, comme la topologie de $F$ est plus fine que celle de $A$ (de la convergence uniforme sur les $\Gamma \cap V_{n}^{0}$ ) ces deux topologies sont identiques et donc $\Gamma^{0}$ est normal dans $F$.

$(2) \Rightarrow(1)$. La topologie de $F$ est alors celle de la convergence uniforme sur les $\Gamma \cap V_{n}^{0}([12]$, V.3.3); on voit alors facilement que l'injection canonique de $F$ dans $A$ est presqu'ouverte au sens de ([11], page 113) et continue; on a donc $F=A$ ([11], page 125).

(2) $\Rightarrow$ (3) est un lemme classique de M. G. Krein ([12],V.3.2).

(3) $\Rightarrow(2)$. Toute $f \in A$ est limite sur $\Gamma$, donc sur $F^{\prime}$ à cause de l'hypothèse (3), d'une suite $\left(f_{n}\right)$ de $F$. Il en résulte que $f$ est bornée sur chaque $V_{p}^{0}$ ([11], page 67-70). L'ensemble $V_{p}^{0}$ est alors $\sigma\left(F^{\prime}, F\right)$ borné, donc absorbé par $\left(\Gamma \cap V_{q}^{0}-\Gamma\right.$ $\cap V_{q}^{0}$ ) pour un certain $q([11]$, page 70$)$. D'où le résultat.

\section{Prolongement d'une mesure conique sur le dual d'un espace de Banach.}

Si $B$ est un espace de Banach, nous allons chercher à prolonger une $\mu \in$ $M^{+}\left(B^{\prime}, B\right)$ en un élément de $M^{+}\left(B^{\prime}, B^{\prime \prime}\right)$. 
Auparavant, rappelons quelques résultats de ([1]), valables dans un cadre plus généal:

11. RAPPELS. ([1], 12-13-14). Avec les notations des préliminaires, soit $\mu \in$ $M^{+}(E, F)$ telle que $K_{\mu} \in E$; soit $E_{1}=R\left(K_{\mu}-K_{\mu}\right)$ et $F_{1}$ le sous-espace de $\left(E_{1}\right)^{*}$ formé des éléments (dont les restrictions sont) continus sur $K_{\mu}$ pour $\sigma(E, F)$; on sait que $\mu$ se prolone canoniquement en $\mu_{1} \in M^{+}\left(E_{1}, F_{1}\right)$ telle que $K_{\mu_{1}}=K_{\mu}$. Cependant, si $\mu$ est localisable dans $E$, par une mesure de Radon généalisée $m$, et que $f \in E^{*}$ soit $m$ intégrable, et que $\left.f\right|_{E_{1}}$ soit dans $F_{1}$, on peut avoir $\mu_{1}\left(\left.f\right|_{E_{1}}\right) \neq$ $m(f)$, comme le montre l'exemple suivant.

12. EXEMPLE. On prend $E=\mathscr{M}([0,1])$ (l'espace des mesures de Radon sur $[0,1])$ et $F=\mathscr{C}([0,1])$ (l'espace des fonctions numériques continues sur $[0,1])$; on prend pour $\mu$ la mesure conique maximale sur $\mathscr{M}^{+}([0,1]) \subset E$ qui représente l'élément $d x$ de $E$; cette $\mu$ est localisable dans $E$ par $m=\int_{0}^{1} \varepsilon_{x}$ et on a

$$
K_{\mu}=\{\varphi d x: \varphi \text { borélienne, } 0 \leqslant \varphi \leqslant 1\} .
$$

Soit $f \in E^{*}$ telle que $f(\pi)=$ (masse de la partie atomique de $\pi$ ); on a $f=0$ sur $K_{\mu}$ et $f=1$ sur le support de $m$; d'où $m(f)=1$ et $\mu_{1}\left(\left.f\right|_{E_{1}}\right)=0$.

13. Proposition. (avec les notations des préliminaires). Soit $\mu \in M^{+}(E, F)$ telle que $K_{\mu} \subset E$, localisable par une mesure de Radon généralisée $m$ sur $(E \backslash 0)$.

Soit $f \in E^{*}$ intégrable pour $m$; les deux propriétés suivantes sont équivalentes.

(1) f appartient à l'adhérence de $F$ dans $L^{1}(m)$.

(2) Pour toutes $m_{1}, m_{2}$ vérifiant $0 \leqslant m_{1} \leqslant m, 0 \leqslant m_{2} \leqslant m$ et $r\left(m_{1}\right)=r\left(m_{2}\right)$ on a $m_{1}(f)=m_{2}(f)$.

Lorsque ces conditions sont réalisées, les deux propriétés suivantes sont équivalentes:

(a) $f$ est continue sur $K_{\mu}$ et pour toute $m^{\prime}$ vérifiant $0 \leqslant m^{\prime} \leqslant m$, qui localise une mesure conique $\mu^{\prime} \leqslant \mu$, le prolongement canonique de $\mu^{\prime}$ à $f$ vaut $m^{\prime}(f)$.

(b) Pour toute $m^{\prime}$ vérifiant $0 \leqslant m^{\prime} \leqslant m$ on a $f\left(r\left(m^{\prime}\right)\right)=m^{\prime}(f)$.

Preuve. L'équivalence de (1) et de (2) résulte du fait suivant: le dual de $L^{1}(m)$ est constitué des différences $m_{1}-m_{2}$, avec $m_{1}, m_{2}$ moindres qu'un multiple (non fixé) de $m$ et le polaire de $F \subset L^{1}(m)$ est formé des différences $m_{1}-m_{2}$ vérifiant $r\left(m_{1}\right)=r\left(m_{2}\right)$.

(a) $\Rightarrow$ (b). Puisque $f$ est continue sur $K_{\mu^{\prime}} \subset K_{\mu}$ le prolongement canonique de $\mu^{\prime}$ à $f$ existe ([1], 12-13-4) et vaut, par définition, $f\left(r\left(\mu^{\prime}\right)\right)=f\left(r\left(m^{\prime}\right)\right)$, d'où (b), car (a) suppose que ce prolongement vaut $m^{\prime}(f)$. 
(b) $\Rightarrow$ (a). Il est clair que (b) $\Rightarrow(2)$, donc (1) est vérifié; il existe donc une suite $f_{n} \in F$ telle que $\int\left|f_{n}-f\right| d m \rightarrow 0$.

On a $\left|\int f_{n} d m^{\prime}-\int f d m^{\prime}\right| \leqslant \int\left|f_{n}-f\right| d m^{\prime} \leqslant \int\left|f_{n}-f\right| d m$, d'où, grâce à l'hypothèse (b),

$$
\left|f_{n}\left(r\left(m^{\prime}\right)\right)-f\left(r\left(m^{\prime}\right)\right)\right| \leqslant \int\left|f_{n}-f\right| d m,
$$

d'où le fait que $f$ est continue sur $K_{\mu}$. Le prolongement canonique de $\mu^{\prime}$ à $f$ vaut, par définition ([1], 12-13-14) $f\left(r\left(m^{\prime}\right)\right)$; comme (b) suppose que cette quantité vaut $m^{\prime}(f)$, le résultat est obtenu.

Dans le cadre classique des mesures de Radon $\geqslant 0$ de masse 1 sur un convexe compact $X$, contenu dans un e.l.c.s., tel que $0 \notin X$, et considéré comme la base du cône $Y$ qu'il engendre, on $a$ :

14. Proposition. Soit $m \in \mathscr{M}_{1}^{+}(X)$ et $f$ une fonction affine sur $Y$, nulle en 0 , telle que $\left.f\right|_{X}$ vérifie le calcul barycentrique pour toutes les $m^{\prime} \in \mathscr{M}_{1}^{+}(X)$, majorées par un multiple (non fixé) de m. Alors $f$ est continue sur $K_{m}$. Si $\left.f\right|_{X}$ vérifie le calcul barycentrique pour chaque $m \in \mathscr{M}_{1}^{+}(X)$, alors $f$ est continue sur chaque $K_{m}$.

Nous allons maintenant chercher à prolonger une mesure conique sur le dual d'un espace de Banach.

15. Proposition. Soit $B$ un espace de Banach qui n'admet pas $l_{1}$ pour facteur direct et $\mu \in M^{+}\left(B^{\prime}, B\right)$ telle que $K_{\mu} \subset B^{\prime}$. Alors $K_{\mu}$ est $\sigma\left(B^{\prime}, B^{\prime \prime}\right)$ compact et $\mu$ se prolonge canoniquement en $\hat{\mu} \in M^{+}\left(B^{\prime}, B^{\prime \prime}\right)$ telle que $K_{\hat{\mu}}=K_{\mu}$.

Preuve. L'espace $H=\left\{\lambda: \lambda \in M\left(B^{\prime}, B\right), \quad \exists k \geqslant 0, \quad|\lambda| \leqslant k \mu\right\}$ s'identifie canoniquement à un espace $\mathscr{C}(K)$, avec $K$ compact et l'application résultante de $H$ dans $B^{\prime}$ est continue, donc, d'après un résultat de Pelczińsky ([10], Théorème 5), comme $B^{\prime}$ ne contient pas $c_{0}$ ([9], page 103$)$, on a $K_{\mu}$ compact pour $\sigma\left(B^{\prime}, B^{\prime \prime}\right)$. D'où le résultat gràce à ([1], 12-13-14).

16. EXEMPLE. Soit $B=l_{1}$ et $\mu \in M^{+}\left(B^{\prime}, B\right)$ telle que $\mu=\sum \varepsilon_{e_{n}}$, où $\left(e_{n}\right)_{n \in N}$ est la base canonique de $B^{\prime}$. Il est clair que $K_{\mu} \subset B^{\prime}$ n'est pas $\sigma\left(B^{\prime}, B^{\prime \prime}\right)$ compact.

17. Proposition. Soit $B$ un espace de Banach séparable qui ne contient pas $l_{1}$; pour toute $\mu \in M^{+}\left(B^{\prime}, B\right)$, localisable en une mesure de Radon généralisée $m$ sur $B^{\prime} \backslash 0$, alors $K_{\mu}$ est une partie $\sigma\left(B^{\prime}, B^{\prime \prime}\right)$ compacte de $B^{\prime}$ et $m$ est portée par un $K_{\sigma}$ de $B^{\prime} \backslash 0$.

De plus, toute $f \in B^{\prime \prime}$ est m-intégrable et si $\hat{\mu}$ désigne le prolongement évoqué en Section 15 , on $a \hat{\mu}(f)=m(f)$. 
Preuve. Elle se fait par étapes:

(a) on a $K_{\mu} \subset B^{\prime}$, d'après la Proposition 4 et $K_{\mu}$ est $\sigma\left(B^{\prime}, B^{\prime \prime}\right)$ compact d'après la proposition précédente. Comme $K_{\mu} \subset B^{\prime}$ et que $B$ est séparable, $m$ est portée par un $K_{\sigma}$ de $B^{\prime} \backslash 0$.

(b) Soit $f \in B^{\prime \prime}$; on va montrer que $f$ est $m$-intégrable; soit $m=\sum_{1}^{\infty} m_{i}$, où chaque $m_{i}$ est fini et portée par une boule de $B^{\prime}$. Comme $l_{1} \not \subset B$ et que $B$ est séparable, on sait que $f$ est de première classe affine sur $B^{\prime}$ et vérifie le calcul barycentrique pour toute mesure de Radon portée par une boule de $B^{\prime}$ ([9], pages 101-102). On a $m\left(f^{+}\right)=\sum_{1}^{\infty} m_{i}\left(f^{+}\right)=\sum_{1}^{\infty} \sup \left(m_{i}^{\prime}(f): 0 \leqslant m_{i}^{\prime} \leqslant m_{i}\right)=\sum_{1}^{\infty} m_{i}^{\prime} \leqslant$ $m_{i}$ d'où $m\left(f^{+}\right)=\sum_{1}^{\infty} f\left(r\left(m_{i}^{\prime \prime}\right)\right)$. Montrons que cette quantité est finie: pour toute $l \in B$ et toute mesure $\pi_{i}$, telles que $0 \leqslant \pi_{i} \leqslant m_{i}$, on a:

$$
\left|\sum_{1}^{n} \pm r\left(\pi_{i}\right)(l)\right| \leqslant \sum_{1}^{n}\left|r\left(\pi_{i}\right)(l)\right| \leqslant \sum_{1}^{n} \pi_{i}(|l|) \leqslant \mu(|l|) .
$$

Comme $B$ est tonnelé, on a: $\left\|\sum_{1}^{n} \pm r\left(\pi_{i}\right)\right\| \leqslant \mathscr{C}$ d'où $\sum_{1}^{\infty} f\left(r\left(m_{i}^{\prime \prime}\right)\right)<\infty$ et $f$ est donc $m$-intégrable.

(c) Pour achever de démontrer lerésultat, on va montrer que $f$ satisfait à la condition (b) de la Proposition 13. Soit $0 \leqslant m^{\prime} \leqslant m$; il faut prouver que $m^{\prime}(f)=$ $f\left(r\left(m^{\prime}\right)\right)$. Soit $m^{\prime}=\sum_{1}^{\infty} m_{i}^{\prime}$ avec $0 \leqslant m_{i}^{\prime} \leqslant m_{i}$; il faut prouver, puisque $m_{i}^{\prime}(f)=$ $f\left(r\left(m_{i}^{\prime}\right)\right)$, que $f\left(r\left(m^{\prime}\right)\right)=\sum_{1}^{\infty} f\left(r\left(m_{i}^{\prime}\right)\right)$. On a vu, à la fin de $\left.b\right)$ que $\left\|\sum_{1}^{n} \pm r\left(m_{i}^{\prime}\right)\right\|$ $\leqslant \mathscr{C}$, d'où $\sum_{1}^{\infty}\left|l\left(r\left(m_{i}^{\prime}\right)\right)\right| \leqslant \infty$ pour toute $l \in B^{\prime \prime}$. D'après ([9], page 103) la série $\sum_{1}^{\infty} r\left(m_{i}^{\prime}\right)$ converge fortement vers $r\left(m^{\prime}\right)$, d'où le résultat.

18. REMARQUeS. (Dans le cadre de la proposition ci-dessus).

(a) $\mathrm{Si} B^{\prime}$ est séparable pour la norme, d'après un résultat de théorie de la mesure ([13], Part I, Chapitre II, Théorème 10, Corollaire 2) la localisation de $\mu$ se fera sur une suite de parties compactes en norme de $B^{\prime}$.

(b) On sait que $B^{\prime}$ peut ne pas être séparable ([7], Théorème 1) et qu'alors il existe dans $B^{\prime}$ une partie discrète pour $\sigma\left(B^{\prime}, B^{\prime \prime}\right)$ et homéomorphe à l'ensemble de Cantor pour $\sigma\left(B^{\prime}, B\right)$.

Le lemme général suivant est utile pour affirmer que certaines mesures coniques sont localisables.

19. LEMME. Soit $E$ un e.l.c.s. de dual $E^{\prime}$ et $H \subset E^{\prime}$ un sous-espace vectoriel qui sépare les éléments de $E$. Soit $\mu \in M^{+}\left(E, E^{\prime}\right)$ telle que $K_{\mu} \subset E$ et soit $\lambda$ la restriction de $\mu$ à $h(E, H)$; si $\lambda$ est localisable en une mesure de Radon généralisée sur $E \backslash 0$, muni de $\sigma\left(E, E^{\prime}\right)$ de la forme $m=\Sigma m_{i}$, où chaque $m_{i}$ est finie et portée par un convexe $\sigma\left(E, E^{\prime}\right)$ compact de $E^{\prime}$, alors $m$ est aussi une localisation de $\mu$.

Preuve. (a) Cas où $m$ est fini et portée par un convexe compact $A$ de $E$. Soit $f \in E^{\prime}$; il existe une suite $\left(h_{n}\right)$ de $H$ qui converge vers $f$ uniformément sur 
$A \cup K_{\mu}$; le résultat est alors évident ([1], 12-13-14) car on a $\mu\left(\left|f_{n}-f\right|\right) \rightarrow 0$ ([3], 30.9) et $\int\left|f_{n}-f\right| d m \rightarrow 0$.

(b) Cas général. Soit $\lambda_{i} \in M^{+}(E, H)$ l'élément localisé par $m_{i}$; comme $K_{\lambda_{i}} \subset$ $K_{\lambda}=K_{\mu}$, cet élément se prolonge canoniquement en $\mu_{i} \in M^{+}\left(E, E^{\prime}\right)$ ([1], 12-13-14); comme $\lambda$ majore toute somme finie de $\lambda_{i}$, on a d'après le (a), $\mu \geqslant \sum \mu_{i}$; l'élément $\nu=\mu-\sum \mu_{i}$ vérifie alors $K_{\nu} \subset K_{\mu} \subset E$ et $\nu=0$ sur $h(E, H)$, d'où $\nu=0$ ([1], 12-13-14) et le lemme est prouvé.

20. EXEMPLes de $\mu \in M^{+}\left(l_{1}, c_{0}\right)$. (a) si $\mu \in M^{+}\left(B^{\prime}, B\right)$, le fait que $K_{\mu} \subset B^{\prime}$ n'implique pas que $\mu$ soit localisable, même si $K_{\mu}$ est compact en norme: on prend $B=c_{0}(N)$ et $B^{\prime}=l_{1}(N)$; soit $\left(f_{n}\right)$ une suite de $L^{1}$ sur $[0,1]$ muni de $d x$, telle que:

( $\alpha) \Sigma\left|f_{n}\right|=\infty$ presque partout.

(B) $\sum \int\left|f_{n}\right|^{2}<\infty$.

( $\gamma$ ) $f_{m}$ et $f_{n}$ sont orthogonales si $m \neq n$.

On peut prendre $f_{n}(t)=(\sin 2 \pi n t) / n$. Pour $t \in[0,1]$, on pose $f(t) \neq\left(f_{n}(t)\right) \in$ $R^{N}$; alors $\lambda=f(d x)$ est élément de $M^{+}\left(R^{N}, R^{(N)}\right)$; montrons que $K_{\lambda}$ est compact en norme dans $l_{1}(N)$ :

On a $K_{\lambda}=\left\{\left(\int g f_{n}\right): g\right.$ borélienne sur $[0,1]$ et $\left.0 \leqslant g \leqslant 1\right\}$. On a

$$
\sum_{p}^{p+q}\left|\int g f_{n}\right|=\sum_{n \in S_{1}} \int g f_{n}-\sum_{n \in S_{2}} \int g f_{n}
$$

où $S_{1}$ et $S_{2}$ forment une partition de $[p, p+q]$; or, si $S \subset N$ est finie, on $a$, si $0 \leqslant g \leqslant 1$ :

$$
\left|\sum_{n \in S} \int g f_{n}\right|=\left|\int_{g}\left(\sum_{n \in S} f_{n}\right)\right| \leqslant\left(\sum_{n \in S} \int\left|f_{n}\right|^{2}\right)^{1 / 2}
$$

ce qui prouve que $K_{\lambda}$ est compact en norme dans $l_{1}(N)$, à cause de $(\beta)$ et $(\gamma)$. On sait ([1], 12-13-14) que $\lambda$ se prolonge alors canoniquement en une $\mu \in M^{+}\left(l_{1}, c_{0}\right)$. A cause de $\alpha$, on ne peut pas localiser $\mu$ sur $l_{1}(N)$.

(b) Si $\mu \in M^{+}\left(l_{1}, c_{0}\right)$ est telle que $K_{\mu} \subset l_{1}$ et vérifie $\sum_{1}^{\infty} \sup \left(\left|x_{n}\right|: x \in K_{\mu}\right)<\infty$ alors $\mu$ est localisable dans $l_{1}$ : En effet, si $\lambda$ est la restriction de $\mu$ en un élément de $M^{+}\left(R^{N}, R^{(N)}\right)$, et si $f_{n}$ est définie sur $R^{N}$ par $f_{n}\left(\left(x_{p}\right)\right)=x_{n}$, on a $([3], 30.9)$ $\sum_{1}^{\infty} \lambda\left(\left|f_{n}\right|\right)<\infty$ et donc $\lambda$, qui est localisable dans $R^{N}$ ([3], 38.8), le sera aussi dans $l_{1}$, d'où le résultat grâce au Lemme 19.

(c) Il existe une $\mu \in M^{+}\left(l_{1}, c_{0}\right)$, localisable sur $l_{1}$, et qui ne vérifie pas la condition de (b).

On la construit, comme en (b), à partir d'une suite $\left(f_{n}\right)$ de fonctions sur $[0,1]$ vérifiant:

( $\alpha) f_{n}(t)=t^{n} / \log n \cdot g_{n}$ où $\left|g_{n}\right|=1$ de sorte que,

( $\beta$ ) $\sum_{p \neq q}\left|\int f_{p} \cdot f_{q}\right|<\infty$. 
On note que $\sum \int\left|f_{n}\right|^{2} \leqslant \Sigma 1 / n(\log n)^{2}<\infty$.

Si $f(t)=\left(f_{n}(t)\right) \in R^{N}$, soit $\lambda=f(d x)$; c'est un élément de $M^{+}\left(R^{N}, R^{(N)}\right)$, localisable dans $l_{1}$, car $\Sigma\left|f_{n}(t)\right|<\infty$; comme en (a) on voit que $K_{\lambda}$ est compact en norme dans $l_{1}$, à cause de $(\beta)$. Soit $\mu \in M^{+}\left(l_{1}, c_{0}\right)$ le prolongement canonique de $\lambda$, tel que $K_{\mu}=K_{\lambda}([1], 12-13-14)$; on a ([3], 30.9): $\sup \left\{\left|x_{n}\right|:\left(x_{p}\right) \in K_{\mu}\right\} \geqslant$ $\sup \left(\int f_{n} \cdot h: h\right.$ borélienne sur $\left.[0,1], 0 \leqslant h \leqslant 1\right)=1 / n \log n$, terme général d'une série divergente. De plus, $\mu$ est localisable dans $l_{1}$ grâce au Lemme 19.

\section{Mesures coniques définissables a partir d'un espace normé}

Soit $E$ un e.l.c.s. de dual $E^{\prime}$; nous allons donner des conditions sur $E$ pour que toute $\mu \in M^{+}\left(E, E^{\prime}\right)$, telle que $K_{\mu} \subset E$, puisse être regardée comme une mesure conique sur un espace normé convenable.

21. Rappels. (1) On dit qu'un espace bornologique convexe ([8]) vérifie la condition de dénombrabilité de Mackey lorsque, pour toute suite $\left(A_{n}\right)$ de bornés, il existe un borné $B$ qui absorbe chaque $A_{n}$.

Si $E$ est un espace de Fréchet, les bornologies suivantes vérifient cette condition ([5], page 156, Théorème 1, page 189, Exercise 1):

(a) La bornologie des parties $\sigma\left(E, E^{\prime}\right)$ bornées.

(b) La bornologie des parties compactes de $E$.

(c) La bornologie des parties faiblement compactes de $E$.

(2) On dit qu'un e.l.c.s. $E$ vérifie la condition stricte de Mackey ([6], Définition 3) si, pour toute partie bornée $A$ de $E$, il existe un disque borné $B$ de $E$ tel que $E_{B}=($ l'espace $R \cdot B$ normé par la jauge de $B$ ) et $E$ induisent sur $A$ la même topologie.

Cette classe d'espaces est stable par sommes directes, sous-espaces, produits dénombrables et contient les espaces métrisables ([5], page 117).

22. Proposition. Soit E un e.l.c.s. qui vérifie la condition stricte de Mackey ( par exemple métrisable $)$ et $\mu \in M^{+}\left(E, E^{\prime}\right)$ telle que $K_{\mu} \subset E$. Il existe alors un espace normé $N$, une $\hat{\mu} \in M^{+}\left(N, N^{\prime}\right)$ telle que $K_{\hat{\mu}} \subset N$ et une injection continue $i$ de $N$ dans $E$ de sorte que $i(\hat{\mu})=\mu$.

Preuve. Soit $A=K_{\mu}$ et $B$ un disque borné de $E$ vérifiant, vis-à-vis de $A$, la condition de (21.2). Soit $N$ l'espace $R \cdot B$, normé par la jauge de $B$. On sait alors ([5], Chapitre 2, Part 8, Exercice (2)) que $K_{\mu}$ est $\sigma\left(N, N^{\prime}\right)$ compact. Par conséquent $\mu$, quipeutêtre considérée comme une mesure conique $\geqslant 0$ sur $N$, mis en dualité avec $E^{\prime}$, se prolonge canoniquement en une $\hat{\mu} \in M^{+}\left(N, N^{\prime}\right)$ telle que 
$K_{\hat{\mu}}=K_{\mu}([1], 12-13-14)$. Il suffit alors de prendre pour $l$ l'injection canonique de $N$ dans $E$.

23. Proposition. (s'applique aux espaces de Fréchet). Soit $E$ un e.l.c.s. qui vérifie la condition stricte de Mackey et dont la bornologie des parties convexes compactes vérifie la condition de dénombrabilité de Mackey.

Pour toute $\mu \in M^{+}\left(E, E^{\prime}\right)$, localisable sur une suite de parties convexes compactes de $E$, telle que $K_{\mu} \subset E$ soit compact, il existe un espace normé $N$, une $\hat{\mu} \in M^{+}\left(N, N^{\prime}\right)$, localisable sur une suite de parties convexes compactes de $N$, telle que $K_{\hat{\mu}} \subset N$ soit compact, et une injection continue $i$ de $N$ dans $E$ telle que $i(\hat{\mu})=\mu$.

24. Proposition. Même énoncé que ci-dessus, en remplacant compact par faiblement compact.

Preuve DE 23. Soit ( $\left.K_{n}\right)$ une suite de convexes compacts de $E$ sur laquelle se fait la localisation de $\mu$ et $A$ un convexe compact de $E$ qui absorbe $K_{\mu}$ et chacun des $A_{n}$. Soit $B$ un disque borné de $E$ vérifiant, vis-à-vis de $A$, la condition de (21.1). Soit $N$ l'espace $R \cdot B$ normé par la jauge de $B$. Comme $K_{\mu}$ est compact dans $N$, il existe ([1], 12-13-14) une $\hat{\mu} \in M^{+}\left(N, N^{\prime}\right)$ telle que $K_{\hat{\mu}}=K_{\mu}$ et qui prolonge $\mu$, considérée comme un élément de $M^{+}\left(N, E^{\prime}\right)$. Cette $\hat{\mu}$ sera localisable, grâce au Lemme 19, sur la réunion des $K_{n}$, qui sont convexes compacts dans $N$. On prendra pour $i$ l'injection canonique de $N$ dans $E$.

Preuve de 24. Pour des parties convexes faiblement compactes, il faut faire appel au résultat de ([5], Chapitre 2, Part 8, Exercice 2) déjà utilisé dans la preuve de la propriété 22 .

25. Remarque. Dans l'énoncé de 23 et de 24 la condition $\mu$ localisable sur une suite, soit $\left(K_{n}\right)$, de parties compactes signifie qu'il existe une localisation, soit $m$, de $\mu$ telle que $\left(0 \leqslant m^{\prime} \leqslant m, m^{\prime}\right.$ finie) entraine $\left(m^{\prime}\right.$ portée par $\left.\cup K_{n}\right)$. Cette condition est à priori beaucoup large que $\left(m=\sum m_{n}, m_{n}\right.$ finie portée par $\left.K_{n}\right)$.

\section{References}

[1] R. Becker, 'Une structure uniforme faible remarquable sur la classe des cônes faiblement complets', Math. Annalen 257 (1981), 447-451.

[2] J. Diestel, Geometry of Banach spaces (Lecture Notes in Mathematics 485 (1975), Springer-Verlag, Berlin, Heidelberg, New York).

[3] G. Choquet, Lectures on Analysis, Vol. 1-3 (Mathematics Lecture Notes Series, Benjamin, New York, Amsterdam, 1969). 
[4] H. Fakhoury et M. Rogalsky, 'Cônes engendrés par un compact étoié ou convexe, applications à l'analyse', Math. Annalen 207 (1974), 47-62.

[5] A. Grothendieck, Topological vector spaces, (Gordon and Breach, New York, 1973).

[6] A. Grothendieck, 'Sur les espaces $F$ et $D \cdot F$, Summa Brasiliensis 3, fasc. 6 (1954), 57-122.

[7] J. Hagler, 'A counter example to several questions about Banach spaces', Studia Mathematica 60 (1977), 289-308.

[8] H. Hogbe Nlend, Théorie des bornologies et applications (Lecture Notes in Mathematics 213 (1971), Springer-Verlag, Berlin, Heidelberg, New York).

[9] J. Lindenstrauss et L. Tzafriri, Classical Banach spaces, Vol. 1 (Ergebnisse der Mathematik und ihrer Grenzgebeite 92 Springer-Verlag, Berlin, Heidelberg, New York).

[10] A. Pelczynski, 'Projection in certain Banach spaces', Studia Mathematica 19 (1960), 209-228.

[11] A. R. Robertson et W. J. Robertson, Topological vector spaces (Cambridge Tracts in Mathematics and Mathematical Physics, Cambridge University Press, 1966).

[12] H. H. Schaeffer, Topological vector spaces, Springer-Verlag, Berlin, Heidelberg, New York, 1971.

[13] L. Schwartz, Radon measures on arbitrary topological spaces and cylindrical measures, Tata Institute, Oxford University Press, 1973.

[14] C. Stegall, 'The Radon Nikodym property in conjugate Banach spaces', Trans. Amer. Math. Soc. 206 (1975), 213-222.

Université Paris VI

Equipe d'Analyse, E.R.A. 294

4 Place Jussieu

75230, Paris

France 